\title{
Gemella morbillorum
}

National Cancer Institute

\section{Source}

National Cancer Institute. Gemella morbillorum. NCI Thesaurus. Code C86892.

A species of facultatively anaerobic, Gram positive, cocci shaped bacteria assigned to the phylum Firmicutes. This species does not form endospores, is oxidase and catalase negative, and non motile. G. morbillorum may be found in the mucous membranes of the oral cavity, upper respiratory tract and the intestines. It is an opportunistic pathogen and a causative agent of endocarditis. 